\title{
Efectos de ser perjudicado o de observar el perjuicio sobre otros en estudiantes universitarios
}

\section{Effects of being harmed or observing the harm on others in university students}

\author{
Ricardo Galguera Rosales ${ }^{\mathrm{a}, *}$, Alejandra Pedraza Herrera ${ }^{\mathrm{a}}$, Alberto de la Rosa Herrera ${ }^{\mathrm{a}}$, \\ Edgar Rocha Hernández ${ }^{a}$, Héctor Octavio Silva Victoria ${ }^{a}$ \\ ${ }^{a}$ Facultad de Estudios Superiores Iztacala, Universidad Nacional Autónoma de México, México
}

\section{Resumen}

Antecedentes: a partir de la caracterización de la conducta su criterio conductual. Objetivo: evaluar el efecto de diferentes distribuciones de perjuicio y la cantidad de este sobre la elección entre perjudicar o no a otro. Método: se empleó un diseño A-B con una línea base y una fase experimental. Participaron 35 estudiantes de educación superior, seleccionados mediante un muestreo no probabilístico por conveniencia y asignados aleatoriamente en alguno de los siete grupos del estudio, diferenciados entre sí por el tipo de distribución del perjuicio (i.e., recibido u observado) y la cantidad de notificaciones sobre este (i.e., 1, 5 y 15), además de un grupo control. La tarea consistió en la resolución de operaciones aritméticas, en la que se simuló la participación de tres compañeros, cuya función podía ser la de perjudicar o ser perjudicado, según fuera el grupo. Resultados: señalan un efecto diferencial significativo entre las distribuciones, así como una tendencia a la reciprocidad cuando el perjuicio es recibido directamente proporcional a la cantidad de este. Conclusiones: son diversas las condiciones multifactoriales de emergencia de la conducta perniciosa como lo son la identificación de quien perjudica, el puntaje, el momento y el sujeto del perjuicio.

Palabras clave: conducta social; conducta perniciosa; conducta socialmente valorada; distribución de perjuicio; notificaciones de perjuicio.

Para citar este artículo:

Galguera, R., Pedraza, A., de la Rosa, A., Rocha, E., \& Silva, H. O. (2021). Efectos de ser perjudicado o de observar el perjuicio sobre otros en estudiantes universitarios. Liberabit, 27(2), e467. https:// doi.org/10.24265/liberabit.2021.v27n2.03 perniciosa entendida como obstaculizar a otro el cumplimiento de

\begin{abstract}
Background: Based on the characterization of harmful behaviors, understood as preventing others the fulfillment of their behavioral criterion. Objectives: This study aimed to evaluate the effect of different harm distributions and amounts regarding the choice of harming others or not. Method: An A-B design was used with a baseline and an experimental phase. Thirty-five (35) higher education students participated in the study. They were selected through a non-probability convenience sampling and randomly assigned to one of the seven study groups or a control group. Such study groups differed from each other by the type of harm distribution -i.e., received or observed- and the number of harm notifications -i.e., 1, 5 and 15-. The task was to solve arithmetic operations wherein the participation of three students was simulated. Said simulated students' role could be to harm or be harmed, according to the group they belonged to. Results: The results indicate a significant differential effect among the distributions. Additionally, a tendency towards reciprocity was shown when someone was harmed, which was directly proportional to the harm amount. Conclusions: It is concluded that there are multifactorial emergency conditions of harmful behaviors, including the identification of the person who harms others, the score, the moment and the harmed person.
\end{abstract}

Keywords: Social behavior; harmful behavior; socially valued behavior; harm distribution; harm notifications.

Este es un artículo Open Access publicado bajo la licencia Creative Commons Atribución 4.0 Internacional. (CC-BY 4.0)

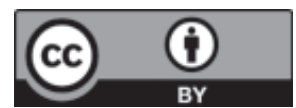

Universidad de San Martín de Porres, Lima - Perú

* ricardo.galguera@iztacala.unam.mx 


\section{Introducción}

El estudio de la conducta social ha generado una vasta cantidad de datos respecto a las diversas funciones de determinados tipos de interacción, descritas regularmente a partir del valor evolutivo de estas. En su momento, Kropotkin (2016) reivindicaba la relevancia de distintos factores, además de la supervivencia del más apto en la explicación darwiniana de la evolución de las especies. Por su parte, Matthews (1966), a propósito del análisis de la agresión entre mamíferos, concluye que existen dos factores que promueven la lucha abierta entre conespecíficos -que puede llevarlos a la muerte-, los cuales están definidos a partir de su valor evolutivo y sus implicaciones para la supervivencia de los organismos: la densidad de la población y la insuficiencia de recursos.

Sin embargo, la conducta social no puede ser reducida ontológicamente a la idea de su valor evolutivo intrínseco ni a los factores que lo determinan, pues la naturaleza del análisis de las dimensiones biológica y social es distinta, a pesar de que la social tenga como sustrato a la biológica. Al respecto, Ribes-Iñesta et al. (2008) precisan que la convivencia, identificada como el descriptor del carácter cultural de la interacción entre individuos, y la supervivencia, entendida como los actos dirigidos a la satisfacción de necesidades filogenéticamente determinadas, deben ser diferenciadas, debido a que esta última «es una categoría aplicable a las especies y organismos biológicos, y constituye un error lógico aplicarla a las culturas» (p. 47). Por esta razón, las formas de comportamiento derivadas del hecho de formar parte de un grupo relativamente estable no pueden ser descritas como expresiones culturales en especies animales no humanas, a menos de que la definición misma de cultura se limitara a entenderla como «una forma de vida compartida por un grupo, [en la que el hecho de] aprender de otros es un requisito fundamental [...]» (De Waal, 2002, p. 38).

Lo descrito hasta aquí supone que se debe distinguir la actividad en grupo de ciertas especies y sus manifestaciones colectivas de la convivencia en términos de convenciones, exclusiva de las relaciones humanas (Ribes, 1992). Fenómenos como el uso rudimentario de herramientas por ciertas especies de primates y su transmisión a través de generaciones; la tendencia de algunas aves por aprender el tono particular de su especie sobre el emitido por otras diferentes; o la aversión aprendida por roedores a alimentos tóxicos mediante la observación directa de conespecíficos (De Waal, 2002), son algunos ejemplos de manifestaciones que involucran la interacción de los miembros del grupo o respecto del grupo y que, no obstante, no son de carácter convencional.

Para entender conceptualmente la distinción entre las dos formas de interacción descritas antes -manifestaciones de carácter biológico/conductas conespecíficas y conducta social-, se debe hacer uso de la categoría de medio de contacto identificada por Kantor (1978) como parte fundamental del modelo de campo. Ribes (2007) acentúa su carácter como posibilitador de contacto entre acontecimientos o eventos a manera de relaciones entre estos, por lo que debe ser entendido como las condiciones que hacen posible una interacción pero que no hacen parte de ella. De esta manera, se identifican tres tipos de medios de contacto que posibilitan las funciones de estímulo-respuesta: fisicoquímico, ecológico y convencional (Ribes \& López, 1985), de los cuales se analizarán los dos últimos. Para Ribes y Pérez-Almonacid (2012), el medio de contacto ecológico consiste en las relaciones que los miembros de una misma especie establecen entre ellos, con otros organismos de diferente especie o con objetos que hagan parte de su espacio geográfico de desarrollo, y se identifica a partir de la supervivencia como posibilidad (Ribes, 2007). Por otra parte, el medio de contacto convencional implica las circunstancias normativas que posibilitan las interacciones determinadas por la sustitución de contingencias mediante conjuntos reactivos convencionales (Ribes, 1990), y es exclusivo de las relaciones humanas. 
Al respecto, Ribes y Pérez-Almonacid (2012) señalan que dichas relaciones no dependen de los individuos particulares, sino de las organizaciones sociales o grupos a los que aquellos pertenecen y están determinadas por las «diferenciaciones compartidas, basadas ya no sólo en atributos físicos sino en funciones de estímulo instituidas» (p. 243). En otras palabras, los sistemas reactivos convencionales dependen tanto de las características situacionales como de la definición compartida de la funcionalidad de la interacción (Carpio et al., 1995). El carácter institucional de las interacciones no constituye una abstracción de la naturaleza de estas, sino que implica interrelaciones prácticas actualizadas en comportamientos acotados a determinadas circunstancia de carácter convencional (Ribes-Iñesta et al., 2008). El medio de contacto convencional se identifica a partir de la convivencia como posibilidad (Ribes, 2007).

En ese contexto, Carpio et al. (1995) reconocen a la dimensión valorativa del comportamiento como parte fundamental del ejercicio de las prácticas convencionales, en tanto implican los criterios de valor que regulan el comportamiento dada cierta comunidad. En principio, tal valoración implica que, a partir de determinados criterios definidos históricamente, algo es juzgado por alguien, sean otros respecto a las relaciones que uno establece, o bien, uno respecto a las relaciones que otros establecen. La práctica de los individuos implica la concreción de la naturaleza socio-convencional de su grupo o grupos de referencia, cuyos criterios de valoración cambian con base en condiciones de distintas dimensiones y a través del tiempo y el espacio, por lo que debe entenderse que no hay criterios de valoración universales (Rodríguez, 2000). En otras palabras, el análisis de las valoraciones no puede llevarse a cabo al margen de las relaciones concretas entre individuos, pues los valores no son entidades abstractas, sino que constituyen un aspecto de las prácticas individuales, enmarcadas en la división social del trabajo (Ribes, 1992).
Por otra parte, Ribes-Iñesta et al. (2008) desarrollan tres dimensiones funcionales que están implicadas en todo sistema de convivencia, cuyos matices están delimitados por las formas específicas que adopta la división social del trabajo y las tareas en cada comunidad según sus determinantes históricos, geográficos, políticos, morales y económicos: las contingencias de intercambio, en las que están implicados los medios de producción y recolección, y dichas relaciones pueden adoptar la función de producción, apropiación o distribución de bienes y servicios; las contingencias de poder, que implican las funciones de prescripción, regulación, administración de consecuencias y el monitoreo o supervisión de las interacciones; finalmente, las contingencias de sanción, que implican las funciones de justificación, autorización o penalización de ciertas formas de convivencia (Ribes, 2001). Cabe aclarar que, en una circunstancia social, estas dimensiones del sistema de convivencia no aparecen de manera aislada ni tampoco puede definirse una correspondencia a priori entre formas de interacción y tipos de contingencia, en tanto que depende de las condiciones específicas de cada interacción. Sin embargo, estos tipos de contingencias y las formas que adoptan en circunstancias dadas pueden manipularse experimentalmente de manera separada (Carranza, 2018; Rangel \& Ribes, 2009; Ribes, 2001).

De esta manera, han sido estudiadas distintas formas que adoptan tales contingencias, como la autoridad total y parcial (Rangel \& Ribes, 2009), la transgresión (Carranza, 2018), el altruismo (Pulido et al., 2015a), la competencia (Pulido et al., 2015b) y la conducta perniciosa (Carpio et al., 2018; Fernández et al., 2018; Martínez et al., 2018; Reyes et al., en prensa; Rocha et al., 2018; Rocha et al., 2021), la cual puede ser considerada como una interacción cuya forma depende de la situación social en la que tiene lugar. Es necesario elaborar una caracterización para mejorar la definición de esta forma que adopta la conducta social, en virtud de lo cual se pueden precisar algunos aspectos bosquejados en trabajos anteriores: a) la conducta perniciosa implica una 
forma de interacción establecida entre dos o más individuos, dados ciertos factores disposicionales; b) dado el carácter ajustivo de las interacciones psicológicas (Kantor \& Smith, 2015), se asume que existen uno o varios criterios a satisfacer en la situación; c) como valoración, se entiende a la conducta perniciosa como la descripción de una afectación negativa al campo contingencial de otro u otros (i.e., contingencias compartidas) mediante comisión u omisión de algún acto, debido a que implica la obstaculización del cumplimiento de un criterio de logro y está bajo el escrutinio de los miembros del grupo apelando al criterio de adecuado o inadecuado (i.e., ajustado o desajustado, según la convención) (Carpio et al., 2018; Rocha et al., 2021); y d) como función de las contingencias de poder y de sanción (Ribes, 2001), implica la identificación de las relaciones que se establecen en determinado grupo y situación social, las cuales pueden adoptar la forma de administración de consecuencias o de penalización, respectivamente.

Se han estudiado diversos factores que promueven la conducta de perjuicio en situaciones específicas, empleándose una tarea estándar que implica la resolución de operaciones aritméticas en el contexto de una interfaz compartida con otros, que en todos los casos han sido simulaciones en computadora. Es decir, los compañeros fueron constituidos por una simulación definida por algoritmos vinculados con el desempeño de los participantes y las variables estudiadas en cada grupo. Este arreglo tiene sentido al asumir que la conducta social no implica, como condición necesaria, satisfacer el criterio de una cantidad determinada de individuos en interacción (Ribes-Iñesta et al., 2008), sino que está definida por las interacciones con valor convencional a partir de intercambios diferidos de contingencias independientemente de si es o no una situación grupal (i.e., $n \geq 2$ ) (Fernández et al., 2018), y representa una alternativa respecto a la preparación metodológica típica de los rompecabezas empleados en estudios de conducta social (Ribes, 2001). En la tarea existe la posibilidad de perjudicar a cualquiera de los tres compañeros -en adelante, compañeros virtuales (CV)- desde el inicio, o bien, en algún momento definido por la condición experimental específica. El perjuicio se lleva a cabo mediante la elección del avatar de alguno de los CV, luego de lo cual aparece una pantalla similar a la de la tarea individual, pero con un color distinto, con la información del CV (i.e., puntaje y avatar). El perjuicio implica la resolución correcta de una operación aritmética a elegir entre las cuatro opciones, luego de lo cual se le resta al contador del CV los puntos que dicha operación tenga predefinidos. Esta preparación experimental, al igual que la propuesta por Ribes (2001), posibilita la elección entre contingencias individuales o contingencias compartidas (PachecoLechón \& Carpio, 2014).

Mediante ese arreglo metodológico, Fernández et al. (2018) evaluaron el efecto de la simetría/asimetría en la dificultad de la tarea sobre la elección de perjudicar o no. Distribuyeron a 27 participantes estudiantes universitarios en tres grupos, cuya característica definitoria fue la dificultad de la tarea (i.e., el rango de las unidades de las operaciones aritméticas) de los CV: 1) más fácil que la propia (i.e., asimetría en contra); 2) igual que la propia (i.e., simetría); y 3) más difícil que la propia (i.e., asimetría a favor). Los autores encontraron que la condición de simetría promovía una mayor frecuencia de conducta perniciosa, mientras que una condición de asimetría a favor implicaba una menor tendencia a perjudicar a los CV. Otro estudio sobre la misma lógica fue el reportado por Martínez et al. (2018), quienes evaluaron el efecto de la simetría/asimetría en la dificultad de la tarea y la ventaja del compañero sobre la elección de perjudicar o no. Participaron 30 estudiantes universitarios quienes fueron asignados de manera aleatoria en tres grupos que se diferenciaban entre sí por la dificultad de la tarea de los CV, como en el caso del trabajo de Fernández et al. (2018). La diferencia radicó en que el puntaje observado en cada uno de los tres CV también se manipuló, esto con base en el criterio de ventaja: 1) más puntos que los propios; 2) mismos puntos que los propios; y 3) menos puntos que los propios. Los resultados de ambos estudios 
coinciden, ya que cuando la tarea del participante es de la misma dificultad que la de los CV (i.e., grupo simetría), existe mayor frecuencia de perjuicio, mientras que hay una tendencia a perjudicar al CV con más puntos que los propios, siendo significativa dicha tendencia solo para el grupo con asimetría en contra. Estos resultados coinciden parcialmente con el planteamiento de Gino y Pierce (2010), quienes explican que algunos comportamientos deshonestos de perjuicio sobre los otros (v.gr., mentir sobre el trabajo del compañero que ganó más para que reduzcan su incentivo y se mantenga la equidad) pueden explicarse a partir de la noción de equidad negativa percibida, es decir, del considerar que se recibe menos que los otros en situaciones grupales respecto a la distribución de los incentivos. El perjuicio, entonces, tiene como uno de sus propósitos el mantener la equidad en la distribución de los incentivos dentro de una organización social.

Otra variable estudiada además de la dificultad -que no es complejidad funcional- y la ventaja en los puntajes, es la forma que adopta la distribución del perjuicio en una situación social. Rocha et al. (2018) evaluaron el efecto de diferentes formas de distribución del perjuicio sobre la elección entre perjudicar o no a un compañero en la misma preparación experimental arriba descrita. Participaron 42 estudiantes universitarios, quienes fueron distribuidos aleatoriamente en siete grupos definidos por la combinación de las siguientes condiciones: a) la identificación del CV pernicioso o la identificación del CV perjudicado; y b) el perjuicio dirigido al participante o a otro CV. La distribución tomó procedimentalmente la forma de notificaciones a los participantes, en las que se indicaba cuál de los CV había perjudicado y a quién había perjudicado, dependiendo del grupo. El estudio constó de una línea base y de una fase experimental, que comenzaba al alcanzar 20 puntos en el contador del participante, y se inauguraba con la primera notificación de un total de 10. En todo momento estuvo habilitada la opción de perjudicar a los CV. Se evidenció un efecto diferencial para cada grupo, así como de las notificaciones de perjuicio. Al respecto, destaca el resultado obtenido en el grupo en el que, además de ubicarse al CV pernicioso, este perjudicaba al participante (se registraron 40 perjuicios), en contraste con el grupo control (con 9 perjuicios registrados). Otro dato que destaca es que el perjuicio de los participantes se dirigió especialmente al CV pernicioso.

Otro estudio realizado a partir de los supuestos bosquejados anteriormente fue el de Rocha et al. (2021), quienes evaluaron el efecto de la exposición a diferentes secuencias de perjuicio sobre la elección entre perjudicar o no. Diseñaron una situación con tres fases experimentales precedidas por su respectiva línea base aplicadas durante tres sesiones distintas. Recuperaron el marco de trabajo del estudio de Rocha et al. (2018), dividiendo a sus 30 participantes en dos grupos, los cuales se diferenciaban entre sí por el tipo de distribución que enmarcaba las secuencias a implementar: 1) $\mathrm{T}$, donde los tres CV perjudicaban; y 2) U, donde un CV identificado perjudicaba. Los dos grupos se subdividieron a su vez en tres, cada subdivisión determinada por la secuencia de perjuicio implementada: a) se perjudica a uno, a mí, a todos -cuya nomenclatura fue, respectivamente, TU, TM, TT para el primer grupo; y UU, UM, UT para el segundo grupo-; b) se perjudica a mí, a todos, a uno -TM, TT, TU; y UM, UT, UU-; c) se perjudica a todos, a uno, a mí -TT, TU, TM; y UT, UU, UM-. Sus resultados señalan que no hubo una diferencia significativa entre las distintas secuencias de perjuicio. Sin embargo, cuando hubo perjuicio, este se dirigió con mayor frecuencia al CV pernicioso, especialmente, cuando este podía ser identificado (i.e., grupos U). Además, el perjuicio de los participantes se dirigió también al CV con mayor puntaje en su contador.

En resumen, se ha estudiado el efecto de las condiciones de simetría/asimetría respecto a la dificultad de la tarea y al puntaje obtenido, así como la distribución del perjuicio y las disposiciones 
derivadas de ello. Sin embargo, es menester ahondar en los valores paramétricos de estas condiciones que ejercen influencia sobre la elección entre perjudicar o no, dicho de otra forma, entre la elección por contingencias compartidas o individuales. En los estudios de Rocha et al. (2018) y Rocha et al. (2021) se emplearon 10 notificaciones de perjuicio en todos los grupos, y se emplearon las mismas distribuciones de perjuicio. Empero, en algunas se obtuvieron datos específicos que permiten destacarlas como distribuciones que promueven, mejor que otras, la conducta perniciosa. Así pues, cabe preguntarse si la variación en la cantidad de notificaciones a manera de información sobre el perjuicio podría tener efectos diferenciales sobre la conducta de perjudicar de los participantes. Por esta razón, el objetivo del presente trabajo consistió en evaluar el efecto de diferentes distribuciones de perjuicio y la cantidad de este, sobre la elección entre perjudicar o no a otro entre estudiantes universitarios al resolver tareas aritméticas. Se espera que existan diferencias entre los grupos en la frecuencia del perjuicio a otros.

\section{Método}

\section{Diseño}

El diseño del estudio fue A-B y constó de dos fases. La primera se trató de una línea base, en la que el participante resolvía las operaciones aritméticas sumando puntos a su contador, mientras observaba el progreso de los CV en sus respectivos contadores. En esta fase, el participante podía perjudicar a cualquiera de los tres CV presionando el botón debajo del avatar correspondiente, pero no recibía alguna notificación de perjuicio. La segunda fue una fase experimental, en la cual aparecía en diferentes momentos una notificación en el centro de la pantalla, deshabilitándose los botones de perjuicio y de selección de la operación aritmética a resolver, lo cual le impedía al participante continuar. Esta fase comenzaba cuando el participante alcanzaba 16 y 28 puntos en el contador, según el grupo experimental. La primera condición se mantuvo vigente para los grupos con cinco y 15 notificaciones, mientras que la segunda correspondió a los grupos con una notificación.

Los participantes fueron asignados aleatoriamente a uno de los siete grupos que constituyeron el estudio, diferenciados entre sí por el tipo de distribución del perjuicio (i.e., el perjuicio está dirigido a otro o va dirigido al participante) y por la cantidad de notificaciones de perjuicio recibidas a lo largo de la fase experimental (i.e., 1, 5 o 15 ocasiones). Así, los grupos fueron los siguientes: a) cuando se identificaba al CV pernicioso (CV $\alpha)$ y al CV perjudicado (CV $\beta)$, y el perjuicio iba dirigido a otro y no al participante (uno perjudica a uno [UU]), así se tuvo al grupo 1) con 1 notificación (1GUU), 2) con 5 notificaciones (5GUU); 3) con 15 notificaciones (15GUU); b) cuando se identificaba al $\mathrm{CV} \alpha$ y el participante era el perjudicado (uno me perjudica a mí [UM]), así se tuvo al grupo 4) con 1 notificación (1GUM), 5) con 5 notificaciones (5GUM), 6) con 15 notificaciones (15GUM); y c) cuando no se perjudicó ni al participante ni a los CV, teniendo así al grupo 7) grupo control sin notificaciones ni perjuicio desde los $\mathrm{CV}$ (SN). En el conjunto de grupos UU se identificaban, entonces, tres roles de los $\mathrm{CV}$ : $\mathrm{CV} \alpha$, $\mathrm{CV} \beta$ y otro $\mathrm{CV}$; en el conjunto de grupos UM se identificaban solo dos roles: $\mathrm{CV} \alpha$ y otro CV. Por otra parte, la frecuencia del perjuicio sobre otros se midió a partir de la cantidad de ocasiones en que el participante presionaba el botón Quitar puntos (ver más abajo la descripción).

\section{Participantes}

Participaron 35 estudiantes de la licenciatura en Psicología de la Facultad de Estudios Superiores Iztacala de la Universidad Nacional Autónoma de México. Sus edades oscilaron entre los 18 y los 23 años. El muestreo realizado fue no probabilístico por conveniencia. El haber participado en algún otro estudio de esta línea de investigación se consideró un criterio de exclusión para la selección de participantes, en tanto estarían familiarizados con la dinámica de simulación que la tarea implicaba. 


\section{Instrumentos}

Se emplearon 20 computadoras de escritorio marca Dell Inspiron con procesador Core i3 y sistema operativo Windows 7 x64 bits, con accesorios individuales como teclado y ratón alámbricos. En cada uno de los equipos se descargó la aplicación elaborada exprofeso para la presente investigación, creada mediante el lenguaje de programación Visual Basic 6.0, así como los archivos requeridos por la aplicación durante el progreso de la tarea. Esta consistió en la realización de operaciones aritméticas básicas (i.e., suma, resta, multiplicación y división), cuya resolución sumaba dos puntos al contador del participante, independientemente del tipo de operación. Así mismo, según fuera el grupo, en el panel inicial se programaban la cantidad de notificaciones recibidas y el tipo de distribución a la que sería expuesto el participante.

Tabla 1

Diseño del estudio

\begin{tabular}{|c|c|c|c|c|}
\hline \multirow{2}{*}{ Grupo } & & \multicolumn{3}{|c|}{ Fases } \\
\hline & & Línea base & Fase experimental & \\
\hline $\begin{array}{l}\text { Uno } \\
\text { perjudica a } \\
\text { uno }\end{array}$ & $1 \mathrm{GUU}$ & \multirow{3}{*}{$\begin{array}{l}\text { Resolución de operaciones } \\
\text { aritméticas. } \\
\text { Sin notificaciones. } \\
\text { Los botones para perjudicar } \\
\text { a los CV están habilitados. }\end{array}$} & $\begin{array}{l}\text { A los } 28 \text { puntos aparece la primera notificación } \\
\text { de perjuicio, que señala al CV } \alpha \text { y al CV } \beta \text {, así } \\
\text { como los puntos restados. }\end{array}$ & \multirow{3}{*}{ 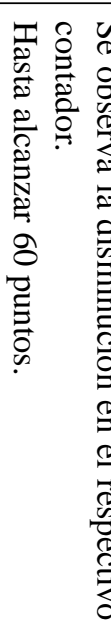 } \\
\hline \multirow[t]{2}{*}{$\begin{array}{l}\text { Uno me } \\
\text { perjudica } \\
\text { a mí }\end{array}$} & 15GUU & & $\begin{array}{l}\text { A los } 16 \text { puntos aparece la primera notificación } \\
\text { de perjuicio, que señala al CV } \alpha \text { y al CV } \beta \text {, así } \\
\text { como los puntos restados. }\end{array}$ & \\
\hline & 15GUM & & $\begin{array}{l}\text { A los } 16 \text { puntos aparece la primera notificación } \\
\text { de perjuicio, que señala al } C V \alpha \text {, así como los } \\
\text { puntos restados al participante. }\end{array}$ & \\
\hline
\end{tabular}

$\begin{array}{lll}\text { Sin perjuicio } & \text { SN } \quad \begin{array}{l}\text { El participante resuelve su tarea hasta alcanzar } 60 \text { puntos. } \\ \text { Sin notificaciones. Los botones para perjudicar a los CV están habilitados. }\end{array}\end{array}$

Nota: Diseño general del estudio, en donde GUU representa a los grupos en los que un CV perjudica a otro CV; GUM a los grupos en los que un CV perjudica al participante; y SN al grupo control, sin notificaciones.

\section{Procedimiento}

La sesión, conducida por los autores de este estudio, se llevó a cabo en una sección de los laboratorios de cómputo de la Unidad de Documentación Científica de la Facultad de Estudios Superiores Iztacala durante el periodo 2020-2, entre los meses de enero y marzo. Cabe mencionar que dicha sección no está abierta a la comunidad universitaria a menos de que se solicite previamente su acceso, por lo que los participantes de la presente investigación estuvieron aislados de los demás usuarios de este servicio que la institución presta a su comunidad. Debido a la cantidad de computadoras disponibles, la sesión se llevó a cabo en dos exhibiciones: la primera con 20 participantes y la segunda con 15. A todos ellos se les invitó formalmente a participar en el estudio y se les acompañó en la lectura del consentimiento informado, previo a la fecha de aplicación de la tarea. 
El tiempo aproximado que les tomó a los participantes concluir su tarea fue de entre 30 y 60 minutos, lo cual variaba según el grupo al que estuvieron asignados. A los participantes se les citó en la entrada del laboratorio de cómputo de la Unidad de Documentación Científica de la FES Iztacala, haciéndolos pasar a alguna de las estaciones de trabajo disponibles. Previo a la llegada de los participantes se abrió y configuró la aplicación, con el propósito de que las condiciones de la tarea ya estuvieran precargadas para cada caso. Luego de tomar sus asientos, se les pedía que atendieran a la explicación que se les daría a continuación, la cual incluía el agradecimiento por formar parte de la investigación, así como la explicación acerca de la naturaleza de la tarea. Se les comentó que trabajarían en una aplicación en línea junto a otros tres compañeros, quienes estarían en la misma sala que ellos distribuidos en alguno de los asientos ocupados en ese momento, así como en otra sección del laboratorio de cómputo. Esto con el propósito de simular la condición compartida de la tarea. También se les explicó que la tarea consistía en la solución a operaciones aritméticas para sumar puntos en sus contadores, concluyendo la actividad al alcanzar los 60 puntos. Se les dijo, además, que tendrían la oportunidad de perjudicar a sus compañeros de tarea en cualquier momento quitándoles puntos, así como de ser perjudicados por ellos.

Se les pidió que completaran los datos de identificación (i.e., clave para el análisis de datos por grupo) y seleccionaran un avatar entre cuatro disponibles. Inmediatamente después de la selección del avatar, el programa simulaba la selección del mote del jugador para cada uno de los CV, dejando disponible solamente el mote P2. Dichos motes fueron P1, P2, P3 y P4, a los que les correspondieron los colores azul, violeta, verde y amarillo. Al seleccionar el mote restante, el participante podía avanzar a la siguiente pantalla, en la que se describían los elementos de la tarea, así como la manera de operar sobre ella.
La pantalla de la tarea mostraba la cantidad de puntos que el participante llevaba acumulados hasta el momento y la cantidad total a obtener (i.e., 60 puntos). Además, mostraba un contador individual debajo de cada uno de los avatares y una sección diferente con el avatar de cada uno de los tres CV junto a un botón con la leyenda Quitar puntos. Para sumar puntos en el contador propio, el participante debía solucionar la operación aritmética elegida. No hubo restricción del tipo de operaciones, por lo que podían elegir siempre la misma o variar entre las cuatro opciones disponibles. En caso de no resolver la operación de manera efectiva, aparecía una etiqueta roja con la leyenda Respuesta INCORRECTA; en caso contrario, es decir, si la resolución era efectiva, la etiqueta que aparecía era de color azul con la leyenda Respuesta CORRECTA, además de que se sumaban dos puntos al contador del participante. En todo momento podía presionar el botón Quitar puntos, correspondiente a alguno de los tres CV para intentar disminuir su puntuación mediante la resolución de una operación aritmética, la cual aparecía en una nueva pantalla de color azul con el avatar del CV al que estuviera buscando perjudicar. El programa registraba la cantidad de ocasiones en que se presionaba dicho botón, así como respecto a qué CV se hacía.

El contador de los CV cambiaba de manera variable con base en dos criterios: 1) un criterio temporal, según fuera la posición del CV en la pantalla, cambiando cada 7, 12 y 10 segundos, respectivamente, condicional a la presión de alguno de los cuatro botones para resolver alguna operación aritmética por parte del participante; 2) un criterio probabilístico, definiendo una probabilidad de .6 de que el contador sumara dos puntos para el respectivo CV en cada ocasión. Lo anterior con el propósito de evitar que coincidiera con los puntos ganados por el participante y mejorar la simulación de estar en una tarea conjunta. Al alcanzar 16 puntos en el contador del participante de los grupos con 5 y con 15 notificaciones, y 28 puntos en los grupos con 1 notificación, en todos los grupos menos en el SN aparecía una notificación de perjuicio justo 
en medio de la pantalla de la tarea, iniciando la fase experimental del estudio. La notificación señalaba el avatar, su mote y color respectivo del CV $\alpha$, así como la cantidad de puntos quitados, el mote y color correspondiente del CV $\beta$. Además, el marco del avatar del CV $\beta$ se iluminaba de color rojo durante los cinco primeros segundos de aparecida la notificación. Para continuar, el participante debía presionar el botón con la leyenda Aceptar que aparecía cinco segundos después de la notificación. Según fuera el grupo, las notificaciones se presentaron una, cinco o quince veces a lo largo de toda la tarea. Las notificaciones aparecieron cuando el contador del participante cambiaba por primera vez, pues en los grupos UM podía repetirse el puntaje en el contador del participante en tanto que se le restaban puntos y debía volver a sumarlos. Al concluir, los participantes recibieron una retribución en especie (i.e., tazas con imágenes alusivas a la Psicología).

\section{Resultados}

Mediante la prueba $H$ de Kruskal-Wallis se compararon los datos de los diferentes grupos, arrojando que existen diferencias significativas entre ellos ( $p=.014$ ), por lo que se rechaza la hipótesis nula que señala que no hay un efecto diferencial de las distribuciones sobre la elección de perjudicar. La comparación post hoc mediante la prueba $U$ de Mann-Whitney arrojó que las diferencias significativas se encuentran entre los grupos $15 \mathrm{GUU}$ y 15GUM $(p=.026)$. En la Figura 1 se representa la dispersión de los datos del perjuicio observado en los siete grupos durante toda la tarea, destacando la tendencia ascendente para el bloque de grupos UM directamente proporcional al aumento en la cantidad de notificaciones, en contraste con el bloque de grupos UU. Del total del perjuicio observado en el estudio, el 64.1\% se concentró en el 15GUM, seguido por el 5GUM (15.2\%) y por el 5GUU (9.8\%), además de que en el grupo control SN se observó un porcentaje mayor de perjuicio (4.3\%) que en 1GUU, 1GUM y 15GUU (3.3\%, 2.2\% y 1.1\%, respectivamente).
Respecto al efecto de las notificaciones, se encontró que en tres de los seis grupos se cometió perjuicio durante la línea base, siendo el porcentaje más alto en el 1GUM. Sin embargo, esto se debió a la cantidad total de perjuicio cometido (i.e., 2 perjuicios en total). Se realizó una prueba de los rangos con signo de Wilcoxon para dos muestras relacionadas, encontrándose diferencias significativas en el perjuicio durante la fase experimental y la línea base $(p=.001)$. Lo anterior confirma que hay un efecto diferencial por presentar las notificaciones en la comisión de perjuicio. Sin embargo, debe considerarse el dato de que el $64 \%$ de los perjuicios cometidos en los grupos UM fueron contingentes a la notificación de perjuicio, mientras que en los grupos UU solo el 8\% del perjuicio cometido fue contingente a la notificación de perjuicio. La Figura 2 muestra las diferencias porcentuales del perjuicio cometido durante ambas fases. En la Figura 3 se muestra cómo se distribuyó el perjuicio cometido por los participantes según el rol de los CV para cada uno de los grupos del estudio. Se llevó a cabo una prueba $Q$ de Cochran, encontrándose diferencias significativas $(Q(2)=$ 78.090, $p<.05$ ) en la proporción de perjuicio cometido hacia los $\mathrm{CV} \alpha$ (67 perjuicios), CV $\beta$ (3 perjuicios) y otro CV (18 perjuicios).

Finalmente, es necesario señalar la relación entre el perjuicio cometido y la cantidad de puntos del CV. Se definieron cuatro criterios con base en los cuales se clasificaron los puntajes de los CV: 1) mejor puntuación, lo cual implica que el puntaje del CV era el más alto de todos, incluyendo al participante; 2) más puntos, pero no mejor puntuación, identificando a los CV con un puntaje superior al participante, pero excluyente de la categoría $1 ; 3$ ) puntos iguales respecto a los del participante; y 4) menos puntos que el participante. Es importante destacar que estos datos se obtenían en cada ocasión que el participante perjudicaba, recuperando su puntaje y el de los CV en ese momento. 


\section{Figura 1}

Distribución de los datos sobre el perjuicio cometido por los participantes de cada uno de los grupos

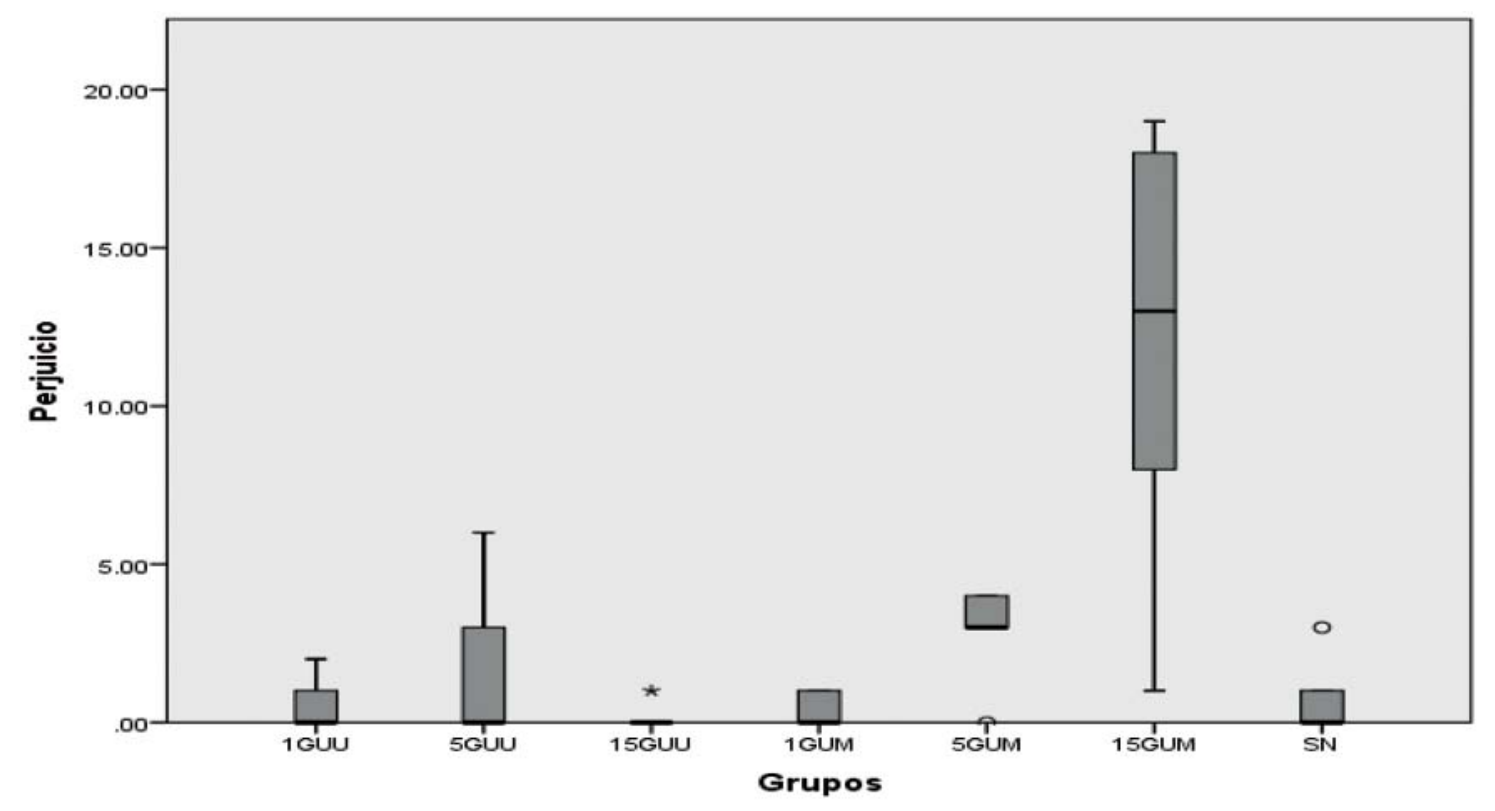

\section{Figura 2}

Comparación del porcentaje de perjuicios cometidos durante la línea base y durante la fase experimental

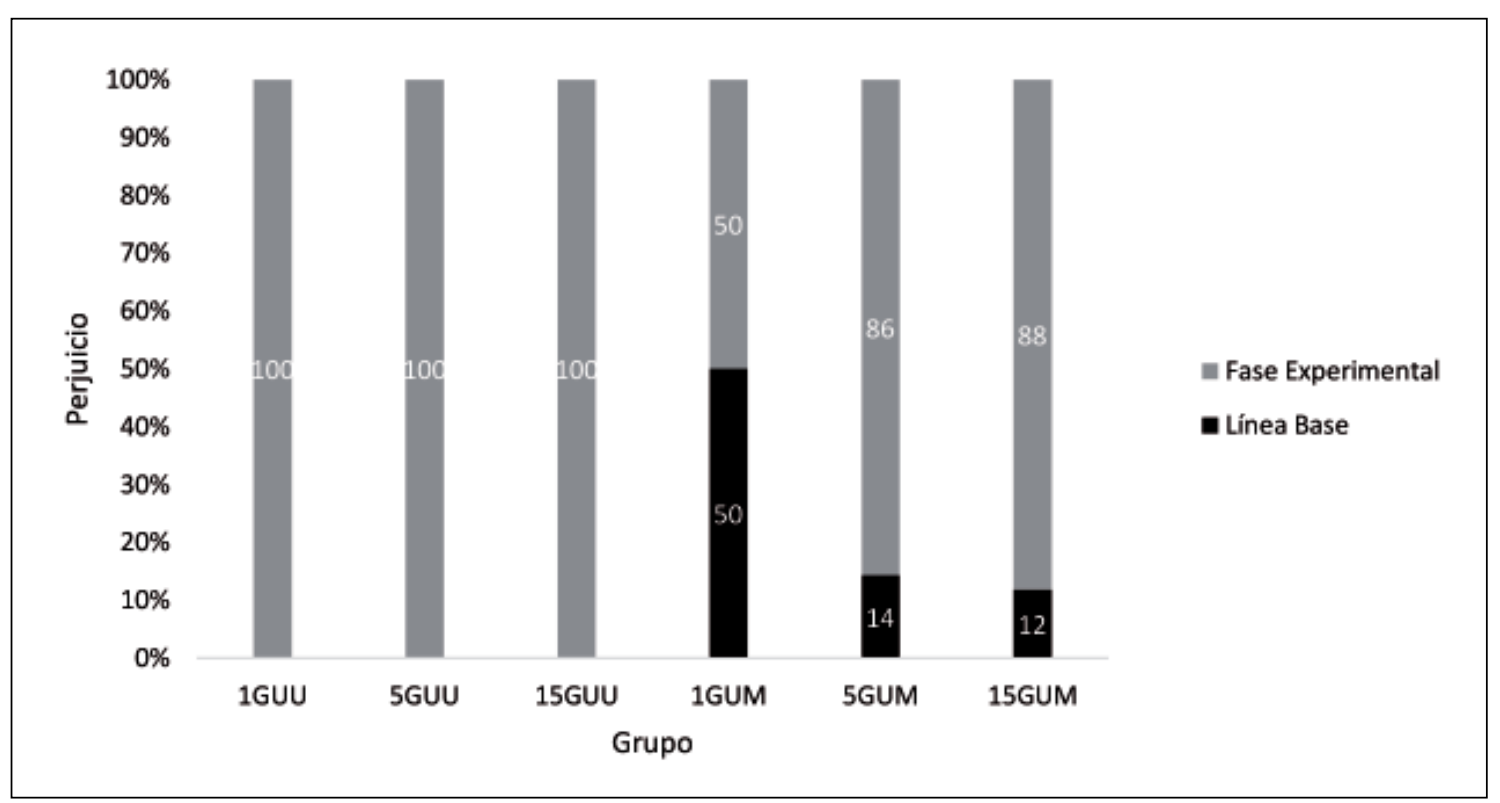




\section{Figura 3}

Distribución de la cantidad de perjuicios cometidos por los participantes de los seis grupos sometidos a las notificaciones

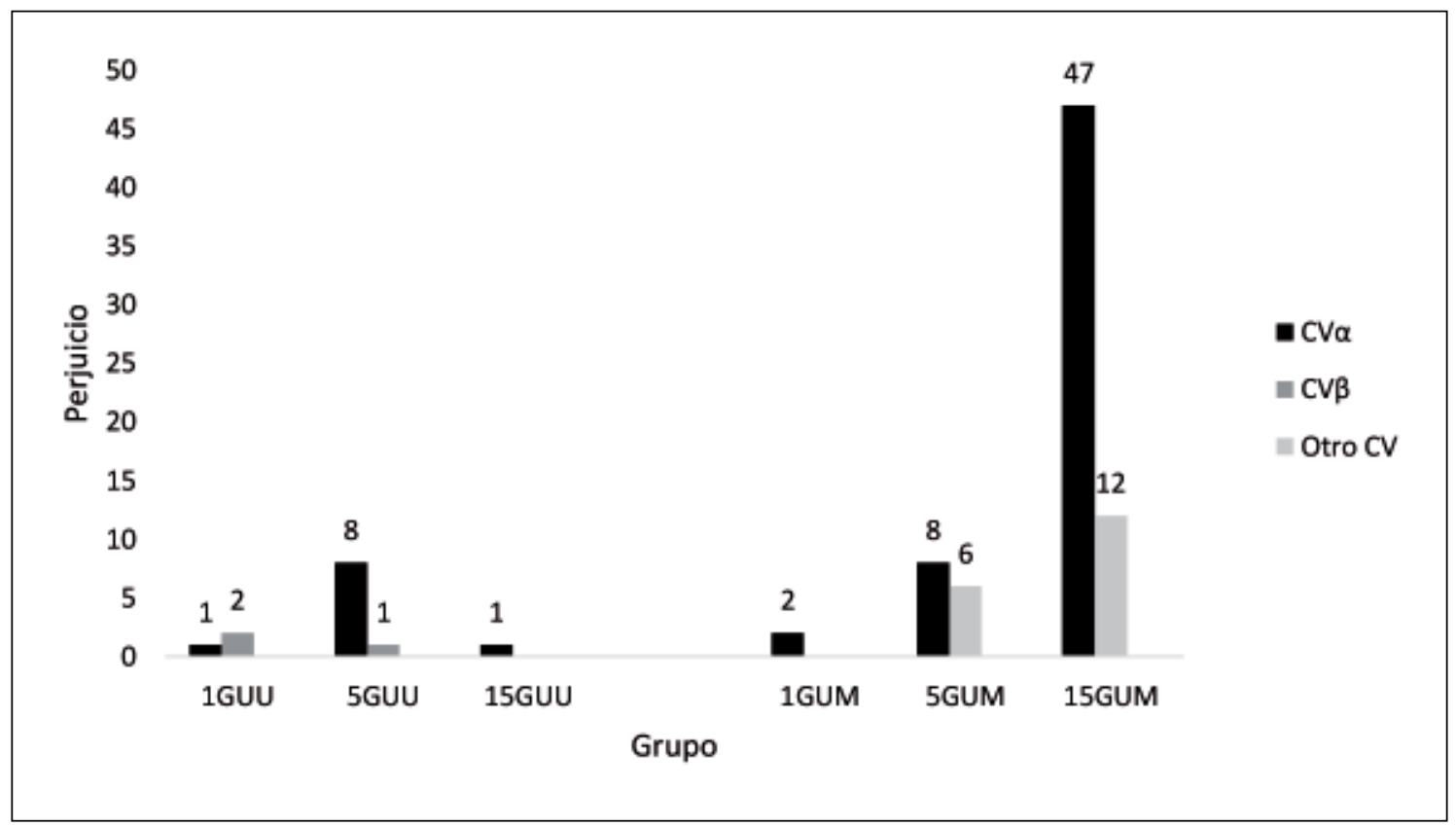

La Figura 4 muestra cómo se distribuyó el perjuicio en el conjunto de grupos UU y UM, es decir, en los grupos 1GUU, 5GUU y 15GUU, por un lado, y 1GUM, 5GUM y 15GUM por otro lado; tomando como criterio para analizarlos en conjunto a la distribución del perjuicio que implicaban (uno perjudica a uno y uno me perjudica a mí, respectivamente). Hubo un efecto diferenciado respecto al perjuicio con base en el puntaje de los CV según pertenecieran a uno u otro conjunto de grupos. Hay que destacar que la cantidad total de perjuicios en los GUU fue de 13, frente a los 75 de los GUM, en los que se pudo observar que el perjuicio se distribuyó entre las cuatro categorías. El 21\% del perjuicio en estos grupos se concentró en los CV con un puntaje superior al del participante, pero no mejor respecto a los demás CV. Es decir, no perjudicaron a aquel CV más próximo a alcanzar los 60 puntos, sino a otro CV. Para analizar lo anterior, en el caso particular del perjuicio dirigido al $\mathrm{CV} \alpha$, se puede señalar que solamente el $30 \%$ de los perjuicios dirigidos a este $\mathrm{CV}$ fueron cometidos cuando este tenía el mejor puntaje respecto a los otros CV y el participante mismo; mientras que el $28 \%$ se dirigió al CV $\alpha$ cuando este tenía más puntos que el participante, pero no era el más próximo a alcanzar los 60 puntos, habiendo otro CV con mayor puntaje al momento del perjuicio. Lo anterior permite indicar que el criterio para perjudicar, al menos en los casos señalados, pudo haber sido otro distinto al de únicamente los puntos obtenidos por los CV. 


\section{Figura 4}

Distribución del perjuicio con base en la puntuación del CV perjudicado para el conjunto de grupos GUU (1GUU, 10GUU y 15GUU) y GUM (1GUM, 10GUM y 15GUM)

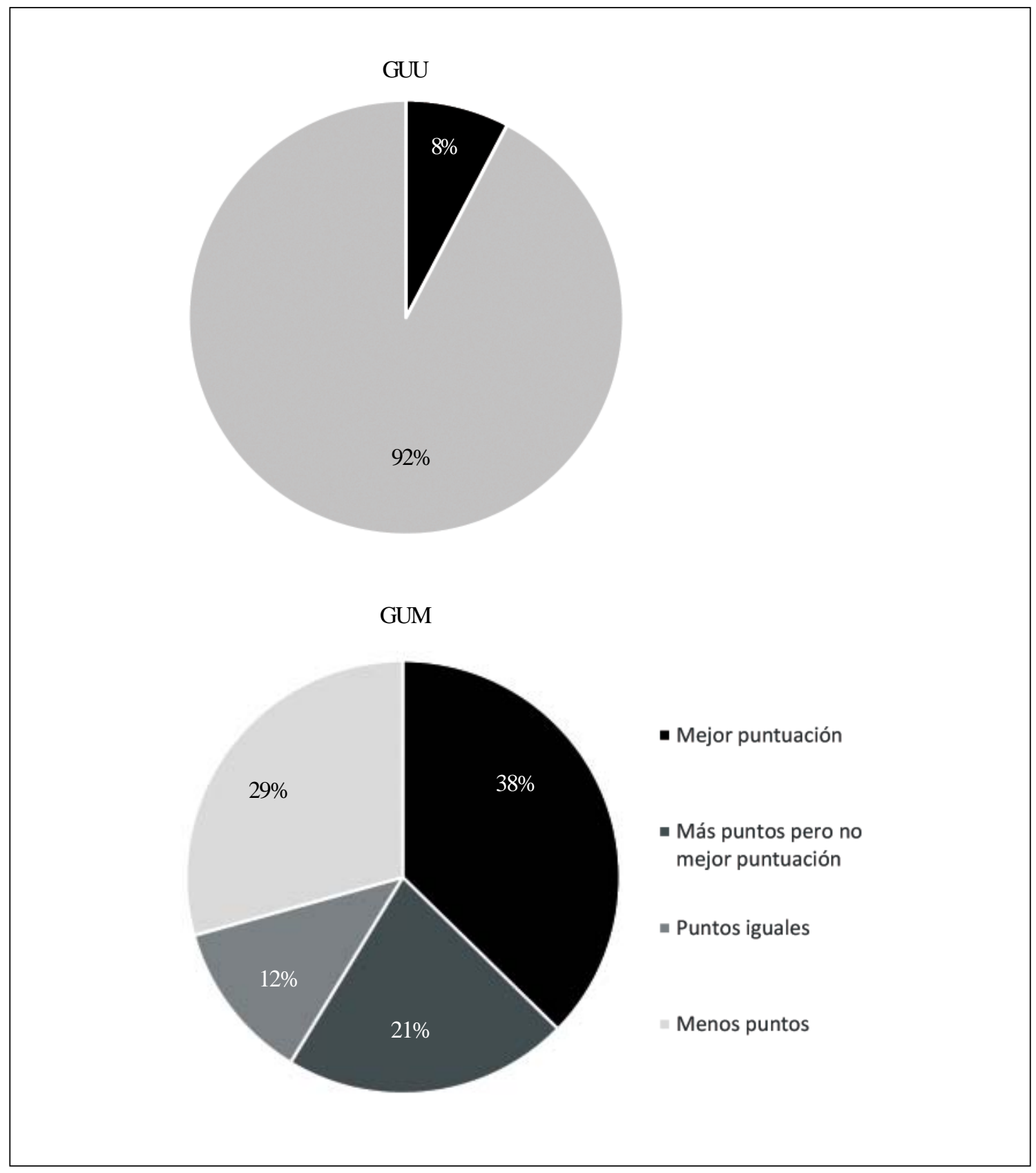




\section{Discusión}

Entre las variables que tienen un efecto sobre la conducta de perjudicar se encuentran la dificultad de la tarea (Fernández et al., 2018; Martínez et al., 2018), la ventaja en los puntajes obtenidos (Martínez et al., 2018), así como la forma que adopta la distribución del perjuicio en una situación social (Rocha et al., 2018; Rocha et al., 2021). Con miras a seguir identificando las variables que promueven la comisión de perjuicio en una situación social, el objetivo del presente estudio consistió en evaluar el efecto de diferentes distribuciones de perjuicio y la cantidad de este, sobre la elección entre perjudicar o no a otro. Se asumía, de principio, que la variación en la cantidad de perjuicios (i.e., las notificaciones de perjuicio) tendría un efecto diferencial en los perjuicios cometidos por los participantes dada la distribución (i.e., recibidos u observados, GUM o GUU, respectivamente).

Se encontró que existe un efecto diferencial de las distribuciones sobre el perjuicio cometido por los participantes, destacando el conjunto de grupos en los cuales el perjuicio se dirigió al participante, especialmente, cuando a este se le notificó en 5 o en 15 ocasiones. La tendencia observada en 1,5 y 15 GUM parece tener continuidad con los resultados reportados por Rocha et al. (2018), quienes encontraron que en el grupo GUM -cuyas características eran iguales a las del presente estudio, excepto por la cantidad de notificaciones presentadas a los participantes que en este caso fueron 10- se perjudicó un total de 40 ocasiones. De forma específica, el orden encontrado en la cantidad de perjuicios entre el estudio de Rocha et al. (2018) y el presente, es el siguiente: 1GUM, 2 perjuicios; 5GUM, 14 perjuicios; 10GUM, 40 perjuicios; 15GUM, 59 perjuicios. Sin embargo, estos resultados no pueden ser del todo generalizados, pues algunas de las condiciones de ambos estudios fueron diferentes.

A partir del análisis de estos datos, parece ser que existe una relación directamente proporcional entre la cantidad de notificaciones de perjuicio y la cantidad de ocasiones en las que el participante perjudica, siempre y cuando sea este al que se perjudica (i.e., UM). Esta tendencia es un indicador de una relación entre la cantidad de los perjuicios recibidos y los cometidos a manera de reciprocidad, entendida en el sentido que Camerer y Fehr (2004) plantean: como disposición, en este caso, a castigar acciones hostiles incluso cuando dicho castigo implique una reducción en la retribución de quien lo hace. En otras palabras, se plantea una correspondencia mutua entre acciones y/o resultados (Ribes \& Pulido, 2015).

A pesar de que el tiempo no constituyó una variable manipulada en el presente estudio, es necesario considerar que los participantes llevaron a cabo la tarea durante su jornada normal de clases. Cabe resaltar el hecho de que para el grupo 15GUM, la duración de la tarea fue ampliamente mayor que para los otros grupos, debido a que, dado sus condiciones específicas, el participante perdía 2 puntos en 15 ocasiones a lo largo de la tarea. Esto implicaba la resolución de 15 operaciones aritméticas adicionales respecto a los participantes del SN y del conjunto de grupos UU -ya que en ellos se perjudicada a otro $\mathrm{CV}$, y no al participante-; 10 operaciones adicionales respecto al grupo 5GUM; y 14 operaciones adicionales respecto al grupo 1GUM. Esto no supone una diferencia en las condiciones experimentales de este grupo respecto a las de los demás, sino solamente el efecto de la condición metodológica a la que, desde el inicio, fue sometido. En ese marco de consideraciones, el $100 \%$ de los participantes del 15GUM perjudicaron a algún CV, siendo el CV $\alpha$ en el $80 \%$ de las ocasiones, llegando así a emplear más tiempo resolviendo operaciones aritméticas para perjudicar a otro que para continuar avanzando en su propio puntaje. Lo anterior coincide con lo planteado por Camerer y Fehr (2004), quienes insisten en que, en condiciones de reciprocidad, el comportamiento está condicionado por las acciones previas de los otros actores de la situación social, y que las consecuencias en las retribuciones del uno no son un factor que modifique la acción recíproca sobre el otro. 
Atendiendo a los datos reportados por Rocha et al. (2018), parece que la cantidad de perjuicios recibidos sí tiene un efecto sobre el establecimiento de relaciones de reciprocidad, siendo más claro este efecto cuando dicha cantidad es mayor. Para el grupo GUM del citado estudio se encontró que el $88 \%$ de los perjuicios cometidos por los participantes se dirigieron al $\mathrm{CV} \alpha$. Lo anterior podría explicar las diferencias en la cantidad total de perjuicios cometidos por los participantes de los grupos 1GUM y 5GUM, pues a pesar de que prevaleció la elección del $\mathrm{CV} \alpha$ para perjudicarlo, la cantidad de perjuicios cometidos fue considerablemente menor. Ribes y Pulido (2015) señalan que las relaciones de reciprocidad son «usualmente personales, intrasituacionales, directas, inmediatas e intracomunidades» (p. 84). De esta manera, las condiciones de cada grupo promovieron que las relaciones de reciprocidad fueran, a su vez, específicas.

Respecto a la demarcación del tópico según las dimensiones funcionales de los sistemas de convivencia (Ribes, 2001), dado el arreglo de la presente tarea, se puede considerar al perjuicio como quitar y/o ser despojado (i.e., de los puntos ganados), de manera similar al caso del altruismo y su respectivo dar y recibir (Pulido et al., 2015a). No obstante, la conducta de perjuicio, aunque conlleva relaciones de intercambio como fundamento, no es expresión de ellas. Al respecto, Ribes y Pulido (2015) mencionan que todas «las relaciones de intercambio se originan en alguna forma de reciprocidad. Sin embargo, no todas las relaciones de reciprocidad involucran relaciones de intercambio» (p. 84). De esta manera se puede señalar que, según sea la situación, el perjuicio es función de las relaciones de poder o de sanción, dada su función de administración de consecuencias ante ciertas formas de comportamiento (v.gr., un individuo da indicaciones equivocadas a otro de cómo trasladarse en una zona que él conoce); o bien, dada su función de penalización consuetudinaria de ciertas formas de interacción (v.gr., restringir los permisos de los hijos para divertirse fuera de casa debido a que no cumplieron con sus deberes del hogar), respectivamente. Se pone especial énfasis al hecho de que la naturaleza de la conducta perniciosa no está definida por una morfología particular (v.gr., golpear, mentir, prohibir, etc.), sino por su función en el sistema de contingencias específico. Se trata, además, de una conducta socialmente valorada, cuyo valor dependerá de las convenciones establecidas históricamente por un grupo (Carpio et al., 1995).

Por otra parte, el criterio con base en el cual perjudicaron al $\mathrm{CV} \alpha$ podría identificarse en su función misma y no solo en los puntajes. Esta suposición está sustentada por el porcentaje de perjuicio cometido hacia el CV $\alpha$ cuando 1) este tenía un puntaje superior al del participante, pero que, a su vez, no era el CV con mejor puntaje en ese momento; 2) cuando tenía el mismo puntaje que el participante; o incluso 3) cuando tenía un puntaje inferior al del participante. A pesar de que, como lo señalan Martínez et al. (2018) y Rocha et al. (2021), la desventaja en la puntuación del participante respecto a la de los CV constituye una variable que promueve la comisión de perjuicio, en el presente estudio se constató que, como fenómeno multifactorial, no es determinante y depende de otros factores en la situación que modifican esta relación, como lo es la condición de recibir el perjuicio o la cantidad de ocasiones en que se recibe. Mediante la recuperación de reportes verbales de los participantes, Rocha et al. (2021) encontraron que, además de perjudicar al CV con la mejor puntuación, otras razones por las cuales cometieron perjuicio fueron la curiosidad y la intención de propiciar el perjuicio entre los otros. Sin embargo, es el corresponder al comportamiento del pernicioso -que autores como Eisenberger et al., (2004) llaman norma de reciprocidad negativa- la principal razón del perjuicio reportado por los participantes (Rocha et al., 2021).

En el presente estudio no se registraron reportes verbales de los participantes respecto a su propia actividad, por lo que se desconocen sus 
verbalizaciones sobre la frecuencia de su perjuicio, lo cual constituye una limitación importante. Por otra parte, a pesar de que la estrategia de simulación respecto al hecho de que cada participante trabajaría con otros tres compañeros ubicados en algún lugar del recinto funcionó -al explicarse la condición simulada al finalizar el estudio, nadie reportó haberlo notado-, es necesario confeccionar situaciones experimentales que impliquen, efectivamente, la acción de varios compañeros -aunque su función sea la de confederados con los objetivos del estudio-, con miras a mejorar la validez ecológica de los estudios sobre conducta social.

$\mathrm{Al}$ igual que en el trabajo reportado por Rocha et al. (2018), en el presente estudio se constató que el porcentaje de perjuicios cometidos fue superior durante la fase experimental, lo cual señala una relación entre observar o recibir perjuicio en una situación social y la comisión de este. A pesar de que en el grupo control SN se cometieron 4 perjuicios, cantidad superior a la cometida en otros tres grupos (1GUU, 15GUU y 1GUM), esto pudo deberse tanto al tipo de distribución (para los grupos UU), como a la cantidad de perjuicios recibidos (para el grupo UM). Cabe destacar que el hecho de que el perjuicio cometido fuera contingente a la notificación de perjuicio, en la mayoría de las ocasiones constituye una fuente para analizar el efecto directo de las variables independientes estudiadas. Con base en lo anterior, al menos se puede concluir que la distribución del perjuicio constituye una variable relevante para su comisión, especialmente, cuando el perjudicado es el participante mismo y, además, que se establece una relación de reciprocidad entre perjuicio recibido y el perjuicio cometido en términos de cantidad. Es decir, mientras más se le perjudique, en más ocasiones perjudicará, siendo aquel que le perjudicó el sujeto del perjuicio. Los resultados obtenidos en esta investigación permiten entender algunas de las condiciones multifactoriales que promueven el perjuicio en estudiantes universitarios, así como plantear la posibilidad de manipularlas en beneficio de la interacción de estos grupos, disminuyendo las condiciones que favorecen su emergencia.

\section{Conflicto de intereses}

Los autores manifestamos que no existe ningún conflicto de interés personal, moral, económico, laboral, de investigación ni cualquier otro respecto al procedimiento de someter el presente manuscrito a evaluación para su posible publicación. Y nos consta que del mismo no puede resultar algún beneficio para los suscritos más que su consideración para ser publicado.

\section{Responsabilidad ética}

Los autores manifestamos que la presente investigación se llevó a cabo atendiendo a cabalidad las prescripciones del Código Ético del Psicólogo publicado por la Sociedad Mexicana de Psicología, velando por el respeto a los derechos y a la dignidad de los participantes. Dado que la naturaleza de la investigación requería la simulación de compañeros virtuales con los que los participantes interactuarían, se procedió conforme al artículo 129 del citado código en el que se señala que las técnicas que impliquen engaño pueden emplearse cuando la prospectiva científica lo justifique. En el presente caso, se requería manipular las relaciones que los compañeros virtuales establecían entre sí y con los participantes. Al finalizar la aplicación, se comunicó a todos los participantes sobre el carácter simulado de la tarea realizada.

A su vez, los autores declaramos que se siguieron los protocolos pertinentes para el cuidado de los participantes. Se siguió la recomendación del Comité de Ética de la Facultad de Estudios Superiores Iztacala respecto a los elementos que la carta de consentimiento informado debía explicitar. Se llevó a cabo, de manera personal, la explicación e invitación formal a participar en el estudio, así como el acompañamiento respecto a la resolución de la tarea y su conclusión, aclarando la naturaleza simulada de la tarea y respondiendo las dudas surgidas. 
Finalmente, los autores declaramos que en el presente estudio no aparecen datos de los participantes ni cualquier otra información que permita su identificación particular.

\section{Contribución de autoría}

RGR: contribuyó con la concepción del estudio y la programación de la tarea. Llevó a cabo la supervisión de la instrumentación del estudio, la recolección de los datos, el análisis estadístico y planteó la estructura general del manuscrito.

APH: contribuyó con la invitación formal a los participantes del estudio, la recolección de los datos y el análisis estadístico. Llevó a cabo la revisión y modificación del manuscrito.

ARH: contribuyó con la invitación formal a los participantes del estudio, la supervisión de la instrumentación del estudio, así como con la recolección y vaciado de los datos. Llevó a cabo la revisión y modificación del manuscrito.

ERH: contribuyó con la concepción del estudio y la discusión del diseño. Llevó a cabo la revisión y modificación del manuscrito final.

HOSV: contribuyó con la discusión del diseño del estudio, así como con el diseño y elaboración de la tarea a realizar. Llevó a cabo la revisión final del manuscrito.

\section{Agradecimientos}

Se agradece el apoyo financiero proporcionado por el proyecto PAPIIT IN306920 de la Dirección General de Asuntos del Personal Académico de la Universidad Nacional Autónoma de México.

\section{Referencias}

Camerer, C., \& Fehr, E. (2004). Measuring Social Norms and Preferences Using Experimental Games: A Guide for Social Scientists. En J. Henrich, R. Boyd, S. Bowles, C. Camerer, E. Fehr, \& H. Gintis (eds.), Foundations of Human Sociality: Economic Experiments and Ethnographic Evidence from Fifteen Small-Scale Societies (pp. 55-95). Oxford University Press. https:// doi.org/10.1093/0199262055.003.0003

Carpio, C., Pacheco, V., Hernández, R., \& Flores, C. (1995). Creencias, criterios y desarrollo psicológico. Acta Comportamentalia, 3(1), 89-98. http://www.revistas. unam.mx/index.php/acom/article/view/18314/17398

Carpio, C., Silva, H., Garduño, H., Pacheco, L., Rodríguez, R., Chaparro, M., Carranza, J., \& Morales, G. (2018). Comportamiento pernicioso: el efecto de las consecuencias en la elección entre perjudicar/no perjudicar a un compañero. Universitas Psychologica, 17(3), 1-12. https://doi.org/10.11144/Javeriana.upsy173.срес

Carranza, J. (2018). El papel de los componentes verbales en la transgresión: efectos de la historia referencial valorativa y las consecuencias [tesis de doctorado, Universidad Nacional Autónoma de México]. http:// 132.248.9.195/ptd2018/noviembre/0782348/Index.html

De Waal, F. (2002). El simio y el aprendiz de sushi. Reflexiones de un primatólogo sobre la cultura. Paidós.

Eisenberger, R., Lynch, P., Aselage, J., \& Rohdieck, S. (2004). Who Takes the Most Revenge? Individual Differences in Negative Reciprocity Norm Endorsement. Personality and Social Psychology Bulletin, 30(6), 787-799. https://doi.org/10.1177/ 0146167204264047

Fernández, A., Galguera, R., Galindo, L., \& Silva, H. (2018). Efectos de la simetría/asimetría en la dificultad de la tarea sobre la conducta perniciosa. Conductual, Revista Iinternacional de Interconductismo y Análisis de Conducta, 6(1), 53-65. https://www.conductual.com/ articulos/Efectos\%20de\%20la\%20simetria-asimetria \%20en\%20la\%20dificultad\%20de\%20la\%20tarea\%20 sobre\%20la\%20conducta\%20perniciosa.pdf 
Gino, F., \& Pierce, L. (2010). Lying to Level the Playing Field: Why People may Dishonestly Help or Hurt Others to Create Equity. Journal of Business Ethics, 95(S1), 89-103. https://doi.org/10.1007/s10551-011-07922

Kantor, J. R. (1978). Psicología interconductual. Un ejemplo de construcción científica sistemática. Trillas.

Kantor, J. R., \& Smith, N. (2015). La ciencia de la psicología. Un estudio interconductual. Universidad de Guadalajara.

Kropotkin, P. (2016). El apoyo mutuo. Un factor de evolución. Editorial Pepitas de Calabaza.

Martínez, D., Olvera, L., Rocha, E., García, A., \& Silva, H. (2018). Asimetría en la dificultad de la tarea y ventaja del compañero en la elección entre perjudicar o no. Enseñanza e Investigación en Psicología, 23(3), 281291.

Matthews, H. (1966). Lucha abierta en los mamíferos. En J. D. Carthy, \& F. J. Ebling (eds.), Historia natural de la agresión (pp. 34-58). Siglo XXI Editores.

Pacheco-Lechón, L., \& Carpio, C. (2014). Mediación lingüística en las interacciones sociales: el caso de las instrucciones y los acuerdos verbales. Revista Electrónica de Psicología Iztacala, 17(2), 695-714. http://www.revistas.unam.mx/index.php/repi/article/ view/47175/42448

Pulido, L., Ribes, E., López, I., \& López, B. (2015a). Interacciones altruistas totales como función de la inducción de reciprocidad. Revista Mexicana de Análisis de la Conducta, 41(1), 32-52. http://rmacmx.org/wp-content/uploads/2015/06/02_RMAC_ 41_1.pdf

Pulido, L., Ribes, E., López, I., \& Reza, A. (2015b). Interacciones competitivas como función de la inducción de reciprocidad. Acta Comportamentalia, 23(4), 359-374. https://www.academia.edu/28031558/In teracciones_competitivas_como_funci\%C3\%B3n_de_la_ind ucci\%C3\%B3n_de_reciprocidad_2015_

Rangel, N., \& Ribes, E. (2009). Leve of Authority and Response Cost in the Obedience of Schoolchildren. Journal of Behavior, Healt \& Social Issues, 1(2), 5365. https://www.redalyc.org/pdf/2822/282221726006.pdf
Reyes, J., Galindo, L., Luevano, S., Rocha, E., \& Olvera, L. (en prensa). Efectos de la historia situacional sobre la elección de contingencias individuales y compartidas. Revista Electrónica de Psicología Iztacala.

Ribes, E. (1990). Psicología general. Trillas.

Ribes, E. (1992). Factores macro y micro-sociales participantes en la regulación del comportamiento psicológico. Revista Mexicana de Análisis de la Conducta, 15(monográfico), 39-55. http://rmac-mx.org/ wp-content/uploads/2013/05/VOL-18-M-39-55.pdf

Ribes, E. (2001). Functional Dimensions of Social Behavior: Theoretical Considerations and some Preliminary Data. Revista Mexicana de Análisis de la Conducta, 27(2), 285-306. http://rmac-mx.org/wp-content/uploads/2013/ 05/Vol-27-n-2-285-306.pdf

Ribes, E. (2007). Estados y límites del campo, medios de contacto y análisis molar del comportamiento: reflexiones teóricas. Acta Comportamentalia, 15(2), 229-259. https://www.redalyc.org/pdf/2745/274520160007.pdf

Ribes, E., \& López, F. (1985). Teoría de la conducta. Un análisis de campo y paramétrico. Trillas.

Ribes, E., \& Pérez-Almonacid, R. (2012). La función lógica del concepto de medio de contacto. Acta Comportamentalia, 20(2), 235-249. https://www. redalyc.org/pdf/2745/274524471008.pdf

Ribes, E., \& Pulido, L. (2015). Reciprocidad, tipos de contingencias sociales sistémicas y lenguaje: investigación de las interacciones interindividuales. Revista Mexicana de Psicología, 32(1), 81-91. https:// www.redalyc.org/pdf/2430/243045363009.pdf

Ribes-Iñesta, E., Rangel, N., \& López-Valadéz, F. (2008). Análisis teórico de las dimensiones funcionales del comportamiento social. Revista Mexicana de Psicología, 25(1), 45-57. https://www.redalyc.org/pdf/ 2430/243016300003.pdf

Rocha, E., Galguera, R., Pedraza, A., Aguilar, F., \& Silva, H. (2018). Distribución del perjuicio como factor disposicional situacional en la elección entre perjudicar o no a un compañero. Revista Electrónica de Psicología Iztacala, 21(4), 1567-1586. http://www. revistas.unam.mx/index.php/repi/article/view/68031/ 60003 
Rocha, R., Olvera-Hernández, L., Díaz-Garduño, A., Basaldúa-Martínez, L., \& Silva-Victoria, H. (2021). Conducta perniciosa: efectos de la exposición a diferentes condiciones de perjuicio. Journal of
Behavior, Health \& Social Issues, 13(1), 50-61. http:// dx.doi.org/10.22201/fesi.20070780e.2021.13.1.68965

Rodríguez, M. (2000). Análisis contingencial. UNAMFESI.

\section{Ricardo Galguera Rosales}

Facultad de Estudios Superiores Iztacala, Universidad Nacional Autónoma de México, México.

Licenciado en Psicología y maestro en Docencia para la Educación Media Superior. Profesor de asignatura «A» en las carreras de Psicología y Psicología del Sistema Universidad Abierta y Educación a Distancia de la UNAM. Sus líneas de trabajo versan sobre las interacciones didácticas y la conducta social.

ORCID: https://orcid.org/0000-0002-7990-0460

Autor corresponsal: ricardo.galguera@iztacala.unam.mx

\section{Alejandra Pedraza Herrera}

Facultad de Estudios Superiores Iztacala, Universidad Nacional Autónoma de México, México.

Licenciada en Psicología y maestrante del programa de maestría y doctorado en Psicología de la UNAM en la residencia de Psicología Escolar. Es coautora de artículos de investigación conductual y ha presentado los resultados de sus trabajos en eventos académicos nacionales e internacionales.

ORCID: https://orcid.org/0000-0002-8097-2592

alepedraza12@gmail.com

\section{Alberto de la Rosa Herrera}

Facultad de Estudios Superiores Iztacala, Universidad Nacional Autónoma de México, México.

Licenciado en Psicología por la FES Iztacala de la UNAM. Actualmente, cursa la maestría en Docencia para la Educación Media Superior en el campo disciplinar de Psicología. Ha colaborado en proyectos de investigación con financiamiento del programa PAPIIT de la DGAPA.

ORCID: https://orcid.org/0000-0002-3663-297X

alberto.dlrhunam@gmail.com

\section{Edgar Rocha Hernández}

Facultad de Estudios Superiores Iztacala, Universidad Nacional Autónoma de México.

Licenciado en Psicología por la FES Iztacala de la UNAM. Profesor de asignatura «A» de la carrera de Psicología de la misma institución. Es autor y coautor de artículos científicos orientados a la enseñanza de la ciencia, el desarrollo del lenguaje, la conducta social, entre otros.

ORCID: https://orcid.org/0000-0001-7003-7560

rochahdez.unam@gmail.com

Héctor Octavio Silva Victoria

Facultad de Estudios Superiores Iztacala, Universidad Nacional Autónoma de México.

Doctor en Psicología del Desarrollo y Educación por la UNAM. Miembro del Sistema Nacional de Investigadores del CONACyT Nivel 1; profesor de Carrera de la FES Iztacala de la UNAM.

ORCID: https://orcid.org/0000-0002-6143-9573

silva.unam@gmail.com 